There is nothing difficult in the preparation of the paper, and a stock of ready-coated drums is a convenience, especially in the rough conditions of largescale field tests.

A. G. Tarrant.

Road Research Laboratory,

Department of Scientific and Industrial Research, Harmondsworth, Middlesex. July 14.

\section{The Polygene Concept}

Dr. Paul G. 'Espinasse voices ${ }^{1}$ misgivings about Mather's polygenic interpretation of selection which are, no doubt, widely felt outside the field of genetics. Each step in the development of genetic theory has given rise to such misgivings. Mather's explanations of his experiments, however, cannot be dismissed as epicyelic (nor for that matter will epicyclic explanations always explain anything). The assumption of polygenes is not even a priori. Hereditary properties are very generally known to show mutation, segregation and linkage. Polygenic variability depends on units, factors, elements, or (why not say it?) genes, which show mutation, segregation and linkage. These are indeed the required criteria. Many important hereditary properties are not individually identifiable. There are hundreds of incompatibility or self-sterility genes known in flowering plants and fungi. Sometimes these genes include only one allelomorphic series, sometimes two. In either case they are not individually but only relationally identifiable. Yet for twenty years no one has questioned the validity of the gene explana. tions of Kniep and East.

Again, in Wnothera there are complex gene differences the linkage, segregation and mutation of which are responsible for the hereditary properties of a large group of species and their hybrids. The genes responsible are located in particular parts of particular chromosomes. No one has estimated their number. No one probably will do so. But that the particles responsible have the chemical constitution, mechanical arrangement and physiological properties recognized in the genes of maize and Drosophila no one doubts.

The "real methodological difficulty" for Dr. 'Espinasse depends on the polygenes being too small in their individual effects to be separated and counted. This was held to be the real methodological difficulty in the way of accepting the atomic theory. It is the kind of difficulty that arises in the making of any hypothesis. It is a difficulty, however, which is habitually relieved by new discoveries. In this case already it can be slightly relieved. Most plants and animals contain in their chromosomes, apart from the ordinary material which is recognized. as carrying the genes of large effect, other parts carrying no identifiable genes. These parts were formerly regarded as inert. Yet they have recently been shown ${ }^{2}$ to exercise an effect on cell processes which influences the character of the whole organism. Their influence is so profound as to be less specific than that of the active genes. They are therefore less readily identifiable. None the less they are the demonstrable object of selection, both natural and artificial; and the numbers of genes in them are quite beyond estimation. Indeed there cannot be much doubt that they fulfil the conditions required by Mather's polygenes. This again is a hypothesis. But it is not an epicyclic hypothesis. On the contrary, it shows that coherence with experimental breeding and with chromosome study which is characteristic of genetic theory.

John Innes Institution,

Merton, S.W.19. July 1.

1 NATURE, 149, 732 (1942).

${ }^{2}$ NATURE, 149, 66 (1942).

MAY I suggest that the dificiculty in respect of the polygene concept, familiar to earlier writers as the theory of multiple or cumulative factors, put forward by Dr. 'Espinasse', is rather logical in character than, as he describes it, methodological. In simple genetics the breeding behaviour exhibited in a group of experiments is 'explained' by a few Mendelian factors, the effects of which are sufficiently different for them to be recognized individually. In the case of many quantitative characters the phenomena of heredity open to observation may be 'explained' on the supposition that there are many Mendelian factors having similar, though not necessarily identical effects. In $1918 \mathrm{I}$ was able to show that such a view accorded well with every feature of the phenomena brought to light by the biometrical study of inheritance in $\operatorname{man}^{2}$. When Dr. 'Espinasse asks "What is the scientific value of such an explanation ?", the answer is the same in both the cases. Its value lies in its ability to predict the results of future breeding operations.

It is revealing to read: "In order to account for the behaviour in heredity of a variable quality ... it seems now to be assumed that its behaviour depends upon the distribution of members of several such pairs, of which there may be no independent knowledge". 'Though the notion is new to him, Dr. 'Espinasse might at least recognize its obviousness and simplicity. The factors postulated are inherited by the same rules which factors investigated independently have been shown to exhibit. They are supposed to be linked in the same systems which have been discovered by the study of individual factors. Does Dr. 'Espinasse find a 'methodological' difficulty in geology when the existence of granite on the surface of the earth leads to the supposition that a similar substance may exist in regions inaccessible to direct observation ?

One of the features to which I directed attention in 1918 is that the hereditary behaviour to be observed does not become more complex as the number of factors is increased. Just as, in the theory of gases the gross behaviour of a molecular aggregate does not depend upon any accidental and momentary configuration of the particles, with its endless complexity, but upon a few constant statistical properties of the aggregate, so it is with the simultaneous action of numerous Mendelian factors. Dr. 'Espinasse's suggestion : "If the number of such pairs invoked as relevant be increased just until they are sufficient to explain the observations", shows a total misapprehension of this situation. In studying the selective response in lint-length of cultivated cottons Panse, for example, has recently exhibited ${ }^{3}$ parallel models, some using few (only three) factors, and others an infinite series. The number of factors involved is in fact one of the least influential features of the systems investigated.

Galton Laboratory,

Rothamsted Experimental Station, Harpenden, Herts.

1 'Espinasse, P. G., NATURe, 149, 732 (1942).

2 Fisher, R. A., Trans. Roy. Soc. Edin., 52, 399 (1918).

a Panse, v. G., Ann. Eugen., 10, 76-105 (1940). 